\title{
Effects of Green Fluorescent Protein or $\beta$-Glucuronidase Tagging on the Accumulation and Pathogenicity of a Resistance-Breaking Lettuce mosaic virus Isolate in Susceptible and Resistant Lettuce Cultivars
}

\author{
Sylvie German-Retana, Thierry Candresse, Emmanuel Alias, René-Pierre Delbos, and Olivier Le Gall \\ Virologie Végétale, IBVM, INRA, BP81, 33883 Villenave d'Ornon cedex, France \\ Accepted 4 November 1999.
}

The RNA genome of a resistance-breaking isolate of Lettuce mosaic virus (LMV-E) was engineered to express the jellyfish green fluorescent protein (GFP) or $\beta$-glucuronidase (GUS) fused to the helper-component proteinase (HC-Pro) to study LMV invasion and spread in susceptible and resistant lettuce cultivars. Virus accumulation and movement were monitored by either histochemical GUS assays or detection of GFP fluorescence under UV light. The GFP- and GUS-tagged viruses spread systemically in the susceptible lettuce cultivars Trocadero and Vanguard, where they induced attenuated symptoms, compared with the wild-type virus. Accumulation of the GFP-tagged virus was reduced but less affected than in the case of the GUStagged virus. Systemic movement of both recombinant viruses was very severely affected in Vanguard 75, a lettuce cultivar nearly isogenic to Vanguard but carrying the resistance gene $\mathrm{mol}^{2}$. Accumulation of the recombinant viruses in systemically infected leaves was either undetectable (GUS-tag) or erratic, strongly delayed, and inhibited by as much as $\mathbf{9 0 \%}$ (GFP-tag). As a consequence, and contrary to the parental virus, the recombinant viruses were not able to overcome the protection afforded by the $m o 1^{2}$ gene. Taken together, these results indicate that GUS or GFP tagging of the HC-Pro of LMV has significant negative effects on the biology of the virus, abolishing its resistance-breaking properties and reducing its pathogenicity in susceptible cultivars.

Additional keywords: infectious cDNA, reporter-tagged potyvirus, viral invasion.

Lettuce mosaic virus (LMV) is one of the most destructive viruses in lettuce and endive crops all over the world (Dinant and Lot 1992). LMV isolates have been classified into four pathotype groups according to their virulence on lettuce varieties carrying the three resistance genes $\mathrm{mol}^{l}, \mathrm{~mol}^{2}$, and $\mathrm{Mo} 2$ identified in Lactuca sativa (Pink et al. 1992a; Bos et al. 1994). The genes $m o l^{l}$ and $\mathrm{mol}^{2}$ are recessive and are believed to be either

Corresponding author: Sylvie German-Retana; Telephone: +33 (0) 556 8432 05; Fax: +33 (0) 5568432 21; E-mail: german@ bordeaux.inra.fr closely linked or allelic (Dinant and Lot 1992). They are deployed worldwide and allow a reasonably effective control of the disease, although resistance-breaking isolates have been reported (Pink et al. 1992b) and constitute a threat to the lettuce industry. Depending on the particular virus isolate/host cultivar combination, the $\mathrm{mol}$ recessive genes result either in resistance (absence of detectable virus multiplication as for example with LMV-1 in $\mathrm{mol}^{2}$ containing cultivars) or in tolerance (systemic virus accumulation but failure to induce symptoms as example with the common isolate LMV-0) (Pink et al. 1992b; Revers et al. 1997a). As for other potyviruses, the genome of LMV consists of a positive-strand RNA of about $10 \mathrm{~kb}$ coding for a single, large polyprotein processed by three virus-specific proteinases (Dougherty et al. 1990; Reichman et al. 1992). The complete sequencing of the genomic RNA of an mol resistancebreaking isolate (LMV-E) and of a common isolate (LMV-0) revealed that there is $97 \%$ amino acid sequence identity between these two isolates regardless of their large differences in pathogenicity, resistance-breaking, and seed-transmission properties (Revers et al. 1997b). Highly infectious cDNA copies of the LMV-E genome have been constructed, with the full-length cDNA placed under the control of the enhanced Cauliflower mosaic virus (CaMV) 35S promoter and of the NOS terminator (Yang et al. 1998).

The jellyfish green fluorescent protein (GFP) and the bacterial $\beta$-glucuronidase (GUS) protein markers have been widely used to elucidate the role of virus-encoded genes and to follow viral invasion of host plants (Dolja et al. 1992; Baulcombe et al. 1995; Casper and Holt 1996). GFP has the advantage of being strongly fluorescent, requiring no substrates or cofactors to form the fluorescent molecule (Chalfie et al. 1994) and thus having proved to be an appropriate, nondestructive technique for the study of the spread of plant viruses during the process of infection (Oparka et al. 1997). Through the use of the reporter-tagged potyvirus Tobacco etch virus (TEV-GUS), the major steps in infection have been compared in resistant and susceptible tobacco plants (Schaad and Carrington 1996). In this study, the genome of LMV-E was engineered to express either the GUS protein or the GFP, fused to the virus helper component proteinase (HC-Pro). The recombinant viruses were then used to study LMV-E infection in the susceptible 
lettuce cultivars Trocadero and Vanguard, and in the resistant cultivar Vanguard 75, which carries the $\mathrm{mol}^{2}$ resistance gene. The results demonstrated that tagging of the HC-Pro with either GUS or GFP significantly affects the accumulation, symptom severity, and resistance-breaking properties of LMV.

\section{RESULTS}

\section{Infectivity and stability of the GUS- or GFP-tagged viruses on susceptible lettuce plants.}

It has previously been shown for TEV that insertion of the GUS gene into the HC-Pro coding sequence immediately downstream of the cleavage site between P1 and HC-Pro does not interfere with the $\mathrm{P} 1$ proteinase processing activity, nor with the viral infectivity (Dolja et al. 1992). Therefore, an equivalent position (3 amino acids downstream of the cleavage site) was used for the insertion of the GFP or GUS genes into the genome of LMV. With an infectious construct in which the full-length cDNA of LMV-E is under the control of an enhanced 35S promoter and of the NOS terminator (Yang et al. 1998), the marker genes were cloned in a suitable AatII restriction site corresponding to the second and third amino acids of the LMV HCPro. The structure of the two tagged LMV recombinants constructed in this way is presented in Figure 1.

Approximately $95 \%$ of lettuce plants (susceptible cultivar Trocadero) inoculated by particle bombardment with the parental plasmid pLMV-E or with the recombinant plasmids pLMVE-GFP and pLMVE-GUS became infected (as judged by the appearance of symptoms). Upon sap inoculation of healthy plants by the viruses derived from plasmid-inoculated plants, the timing of the systemic infection process was found to be the same for LMV-E and LMV-E-GFP, whereas a 2-day delay was generally observed in the case of LMV-E-GUS.

Aphid transmission experiments indicated that LMV-E-GFP was no longer aphid transmissible (not tested in the case of LMV-E-GUS), a result that parallels the observation that TEV-GUS also lost its aphid transmissibility (Dolja et al. 1992). However, a recombinant TEV tagged in a similar fashion with a polyhistidine track still retains this property (Blanc et al. 1999) and thus it is more likely that it is the size or nature of the domain fused to the HC-Pro N-terminal end that affects aphid transmissibility rather than the mere fact that this region has been modified.

The stability of the tagged recombinants was tested in two kinds of experiments. In the first experiment, serial passages were performed every 2 weeks (GUS-tag) or every month (GFP-tag) while in the second experiment, conducted in parallel, back inoculations were performed at timed intervals from the same plant, to test the effect of a prolonged propagation of the tagged viruses on the stability of the insert. The transfer of LMV-E-GFP from plant to plant at monthly intervals resulted in very stable retention of GFP expression: even following six consecutive passages, GFP was detected in all inoculated plants. In contrast, prolonged propagation for 4 months in the same host plant resulted in the recovery of viral isolates having lost the ability to express GFP fluorescence. In the case of the GUS-tagged virus, recovery of isolates having lost the ability to express GUS activity was observed earlier. Indeed, such isolates were recovered within 3 months, either in a given plant after prolonged infection or after a few 2week serial passages. The GFP insert in the LMV-E genome therefore appears to be significantly more stable than the GUS insert in the LMV or TEV genomes (Dolja et al. 1992).

\section{Analysis of deleted variants derived from the GUS- or GFP-tagged viruses.}

The cDNA spanning the region of insertion of the GFP or GUS genes in the recombinant viruses having lost reporter gene activity (hereafter named respectively LMV-E- $\Delta$ GFP or LMV-E- $\Delta$ GUS) was amplified by reverse transcriptionpolymerase chain reaction (RT-PCR) from 10 individual plant samples. In all cases, a single, amplified fragment was obtained. Following sizing on agarose or polyacrylamide gels and restriction enzyme digestion analysis, it was demonstrated that in all cases loss of the activity of the reporter gene was associated with deletions in the introduced sequence as well as in the adjoining HC-Pro coding sequence. Four amplification products were selected and sequenced to map the deletion endpoints. Two products (LMV- $\Delta$ GFP1 and $-\Delta$ GFP3) were found to be identical in sequence and, therefore, are likely to derive from the same deletion event. Figure 2 shows that these four sequences analyzed lacked nucleic acid sequences encoding either 24 or 108 amino acids from the $\mathrm{NH}_{2}$-terminal part of the HC-Pro and retained between 1 and 40 amino acids of the GFP protein. The P1 coding region was unaffected in all of the deletion mutants analyzed. As expected for a virus expressing its genome as a polyprotein, all mutants maintained the genomic open reading frame. For the GUS deletion mutants, only LMV-E- $\Delta$ GUS3, which showed the largest deletion as estimated from the size of the RT-PCR amplified, was sequenced. This mutant lacked the entire inserted sequence and had the largest deletion we could detect in the HCcoding region (109 amino acids).

\section{Symptoms and accumulation of the tagged viruses on susceptible butterhead lettuce cultivar Trocadero.}

The susceptible butterhead lettuce cultivar Trocadero inoculated with LMV-E-GFP or with LMV-E-GUS developed attenuated symptoms, compared with plants inoculated with LMV-E (Fig. 3). Typically, LMV-E first induces a strong vein clearing followed by the development of a severe stunting of the plants accompanied by leaf deformation and a mosaic with occasional necrosis (Fig. 3A,B). Both the GFP- and GUS-tagged viruses induced a somewhat less intense vein clearing than the parental virus. In the case of LMV-E-GUS, appearance of this initial symptom was, on average, delayed by 2 days. The plants inoculated with the two tagged viruses then displayed typical mosaic symptoms (slightly more severe with LMV-E-GUS) but failed to exhibit the severe stunting/leaf deformation or the necrotic reactions observed on LMV-E-inoculated plants (Fig. 3C,D). In LMV-E-GFP-infected lettuce, the fluorescence was completely superimposable to the vein clearing symptoms and, in leaves developed later on, to the yellow-green areas of the mosaic pattern (Fig. 4A,B). Therefore, the tagging of the HCPro of LMV with either GUS or GFP appears to have a negative effect on the pathogenicity of LMV-E on the susceptible butterhead cultivar Trocadero. The symptoms of the GFP or GUS deletion mutants were usually comparable to those of the tagged viruses and were clearly attenuated, compared with the wildtype LMV-E virus (not shown).

The levels of accumulation of the tagged viruses and of some of the deletants were evaluated by semiquantitative en- 
zyme-linked immunosorbent assay (ELISA) and compared with the accumulation of wild-type LMV-E at 15 days and 30 days post inoculation (dpi). As shown in Table 1, significant differences were observed both with the wild-type virus and between the two tagged viruses. LMV-E-GFP accumulated to a level comparable to that of LMV-E at 15 dpi but showed a clearly reduced accumulation at $30 \mathrm{dpi}$. In contrast, the accumulation of LMV-E-GUS was more significantly impaired, reaching only 26 and $6 \%$ of that of LMV-E at 15 and 30 dpi, respectively. The deletion mutants accumulated to levels slightly higher (LMVE- $\Delta$ GUS3) or lower (LMV-E- $\Delta$ GFPs) than their parental tagged GUS or GFP virus at 30 dpi in Trocadero (Fig. 5).

\section{LMV-E}

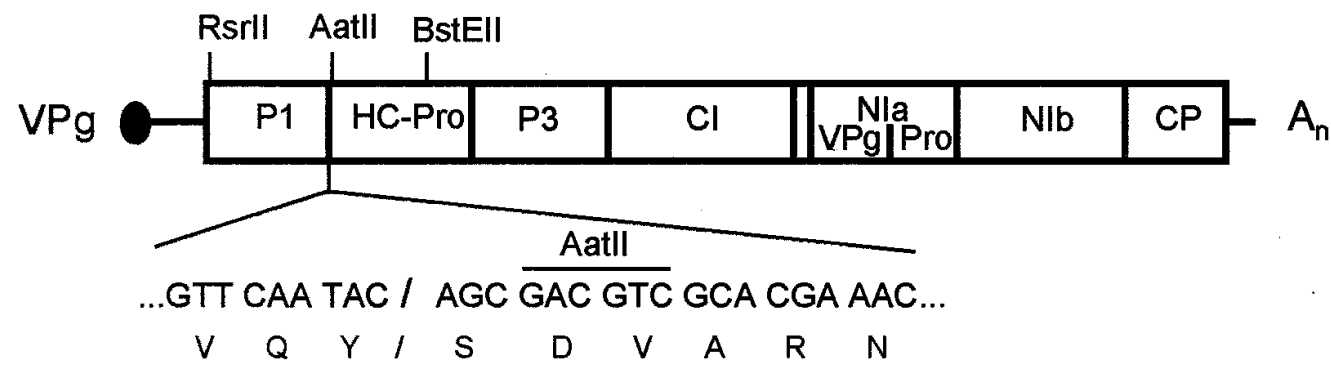

LMV-E-GFP

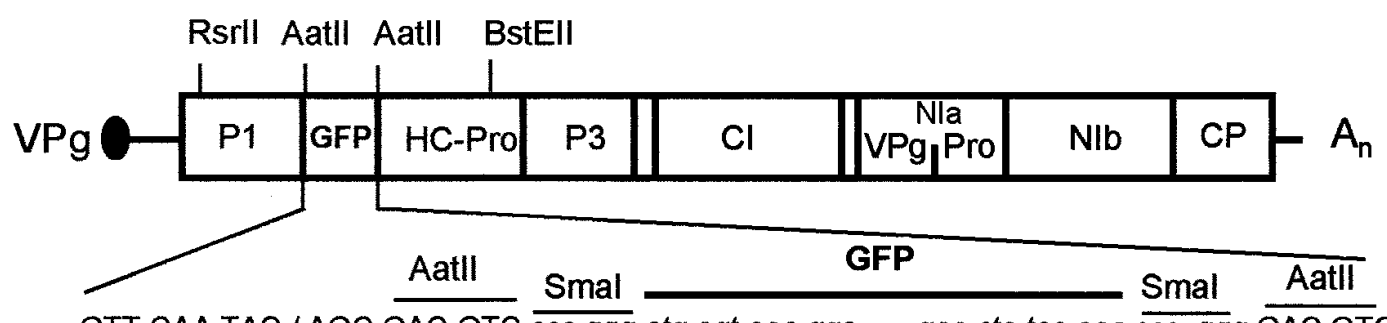

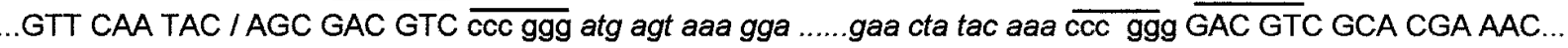

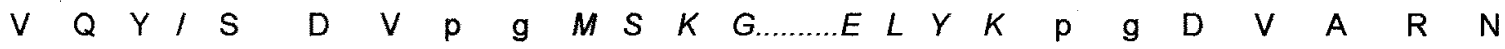

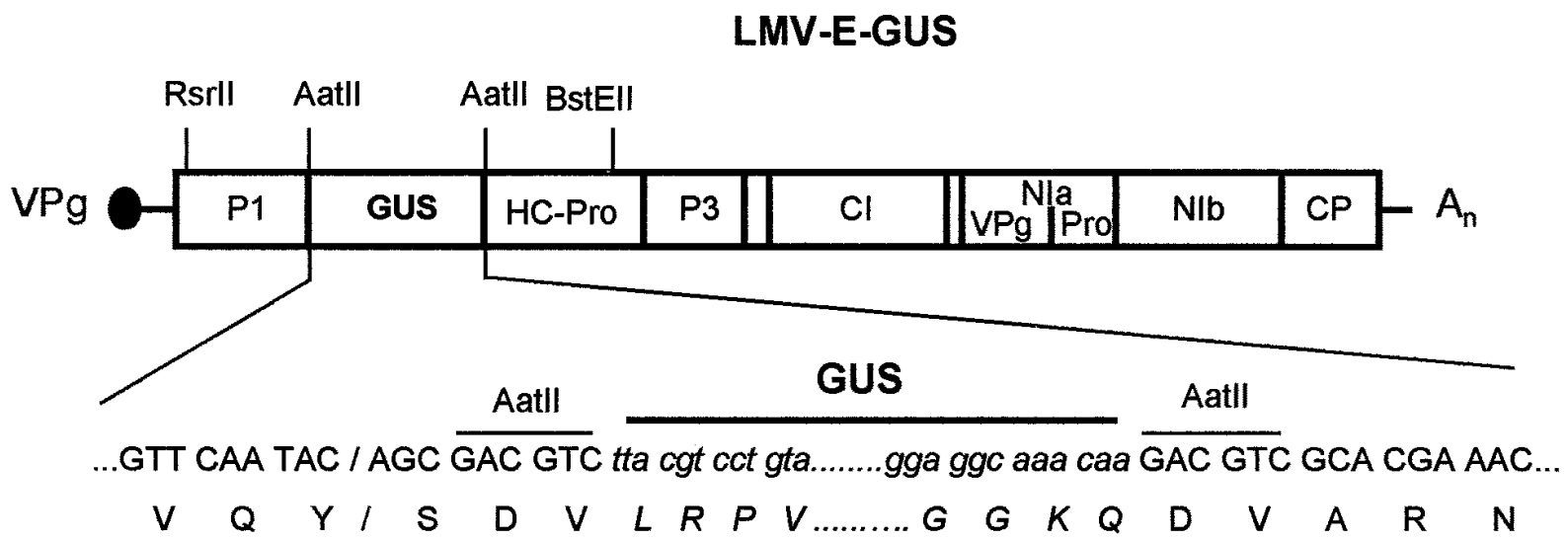

Fig. 1. Schematic representation of Lettuce mosaic virus resistance-breaking isolate (LMV-E) genome and tagged (green fluorescent protein, GFP; $\beta$ glucuronidase, GUS) recombinants. Nucleotide and amino acid sequences modified in LMV-E-GFP and LMV-E-GUS with respect to wild-type LMV-E are indicated below each diagram. Restriction endonuclease sites used for subcloning are shown above diagrams. Nucleotide sequences specifying restriction endonuclease recognition sites are indicated. Sequences corresponding to the reporter genes are indicated in italics. The genome organization of LMV is indicated for each construct. 
In summary, GFP or GUS tagging of the HC-Pro of LMV-E resulted in viruses showing less severe symptoms in the susceptible butterhead cultivar Trocadero and with slightly (GFP-tag) or severely reduced (GUS-tag) ability to accumulate in systemically infected leaves, especially at later infection times. The overall pathogenicity of the recombinant viruses was thus affected by these modifications. These results support those of Guo et al. (1998), who showed that the incorporation of the GUS gene into the Plum pox virus (PPV) genome affects the ability of this potyvirus to infect Nicotiana clevelandii plants.

Symptoms and accumulation of the tagged viruses in iceberg lettuce cultivars differing by the presence of the $m o 1^{2}$ resistance gene.

To analyze the effects of the $m o l^{2}$ tolerance/resistance gene on the GFP- or GUS-tagged viruses, a pair of closely related iceberg lettuce cultivars, Vanguard and Vanguard 75, was selected. Vanguard 75 was derived from Vanguard by introgression of the $\mathrm{mol}^{2}$ resistance gene followed by six consecutive backcrosses to recover a genotype as close as possible to the original Vanguard type (Ryder 1979). Thus, although not strictly isogenic for the resistance gene, these two cultivars are very closely related and the presence of the $\mathrm{mol}^{2}$ gene is expected to be the major difference between them.

Similar to Trocadero, the susceptible cultivar Vanguard displayed severe symptoms when infected by LMV-E (not shown). Systemic invasion of the plants was rapid, with symptoms usually visible by 7 to 9 days following sap inoculation. The symptoms initially included vein clearing, followed by necrotic mosaic and severe stunting. In contrast, and although LMV-E is a resistance-breaking isolate, systemic invasion of the resistant cultivar Vanguard 75 was considera-

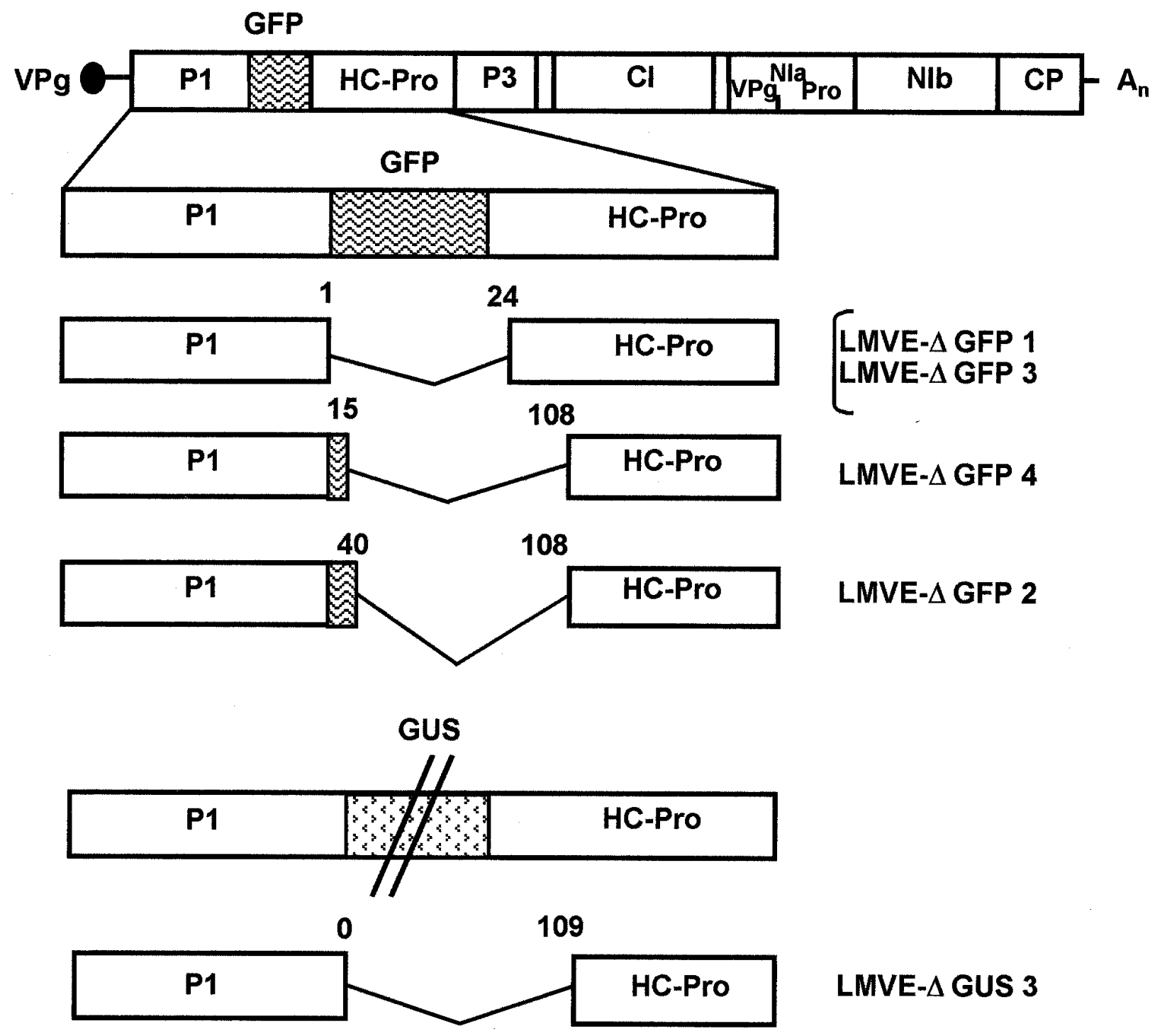

Fig. 2. Genetic map of Lettuce mosaic virus resistance-breaking isolate (LMV-E) deletion variants. Sequences missing from various deletion variants of LMV-E-GUS and LMV-E-GFP analyzed are shown. Numbers indicate amino acid positions of the deletion endpoints in the green fluorescent protein (GFP), $\beta$-glucuronidase (GUS), and helper-component proteinase (HC-Pro) sequences in the spontaneous mutants (e.g., in LMV-E- $\Delta$ GFP4 the GFP amino acid 15 is present but the HC-Pro amino acid 108 is absent). In LMV-E- $\Delta$ GFP1 and LMV-E- $\Delta$ GFP3, only the N-terminal methionine of GFP remains, while in LMV-E- $\Delta$ GUS3 all inserted sequences, including the additional AatII and SmaI sites, have been removed. 
bly slower, systemic movement and symptoms being first detectable about 3 weeks post inoculation (not shown).

In Vanguard, the tagged viruses behaved essentially as in Trocadero: early systemic movement (with a 2-day delay for LMV-E-GUS), and strongly attenuated symptoms (not shown). Accumulation of the tagged viruses reached levels comparable to those observed in Trocadero by $30 \mathrm{dpi}$. However, viral accumulation at $15 \mathrm{dpi}$ in Vanguard reached only about $10 \%$ of the respective levels observed in Trocadero (Table 1). Altogether, these results thus confirm and extend to a different lettuce type, iceberg, the reduced pathogenicity observed for the tagged viruses in the butterhead cultivar Trocadero and in addition they indicate that the kinetics of viral accumulation differ between the iceberg cultivar Vanguard and the butterhead cultivar Trocadero.

Neither LMV-E-GFP nor LMV-E-GUS (nor any of the deletion mutants) was able to induce detectable symptoms in the resistant cultivar Vanguard 75, up to the end of the experiments ( 2 months post inoculation). GUS staining or ELISAs failed to reveal any systemic movement of LMV-E-GUS, at any time point, except for two plants that displayed GUS activity 3 weeks post inoculation in one particular experiment. However, histochemical staining of inoculated leaves demonstrated that LMV-E-GUS is able to multiply and move cell to cell in this host because infection foci ( 2 to $3 \mathrm{~mm}$ in diameter) were readily detected 7 to 13 dpi (Fig. 4D). Detailed analysis of the number of blue cells in the lesions on inoculated leaves at 36,48 , and $72 \mathrm{~h}$ post inoculation revealed that viral cell-tocell movement was significantly slower in Vanguard 75 than in Vanguard. At all time points a statistically significant difference was detected between the number of blue cells per lesion observed in Vanguard, compared with Vanguard 75, with, at $48 \mathrm{~h}$ post inoculation, $20 \pm 9$ and $5 \pm 2$ blue cells per lesion, respectively (Fig. 4E,F).

LMV-E-GFP was occasionally, but more often than LMVE-GUS, found to be able to move systemically in Vanguard 75. However, this systemic movement was very erratic and only observed on a limited number of plants and not for each experiment performed. In addition, when occurring, this movement was extremely slow, usually becoming detectable as low levels of fluorescence with a very limited distribution on the leaf blades only at 5 to 6 weeks post inoculation (Fig. $4 C)$. Fluorescence in these areas never increased in intensity or in distribution upon further observation (not shown). The accumulation level of LMV-E-GFP in those few plants showing evidence of systemic movement ultimately reached around $13 \%$ of that of the LMV-E control at 2 months post inoculation (Table 1).


Fig. 3. Symptoms induced by Lettuce mosaic virus resistance-breaking isolate (LMV-E), LMV-E-GUS ( $\beta$-glucuronidase), and LMV-E-GFP (green flurorescent protein) on the lettuce cultivar Trocadero. A, Trocadero plants infected with LMV-E (left), LMV-E-GFP (center), and LMV-E-GUS (right) at 20 days post inoculation (dpi). B, Trocadero plant infected with LMV-E at 20 dpi, showing characteristic severe stunting caused by this isolate. Comparison of symptoms induced on systemically infected leaves of Trocadero at 20 dpi by (C) LMV-E (two leaves on left) and LMV-E-GUS (right) and (D) LMV-E (two leaves on left) and LMV-E-GFP (right). 

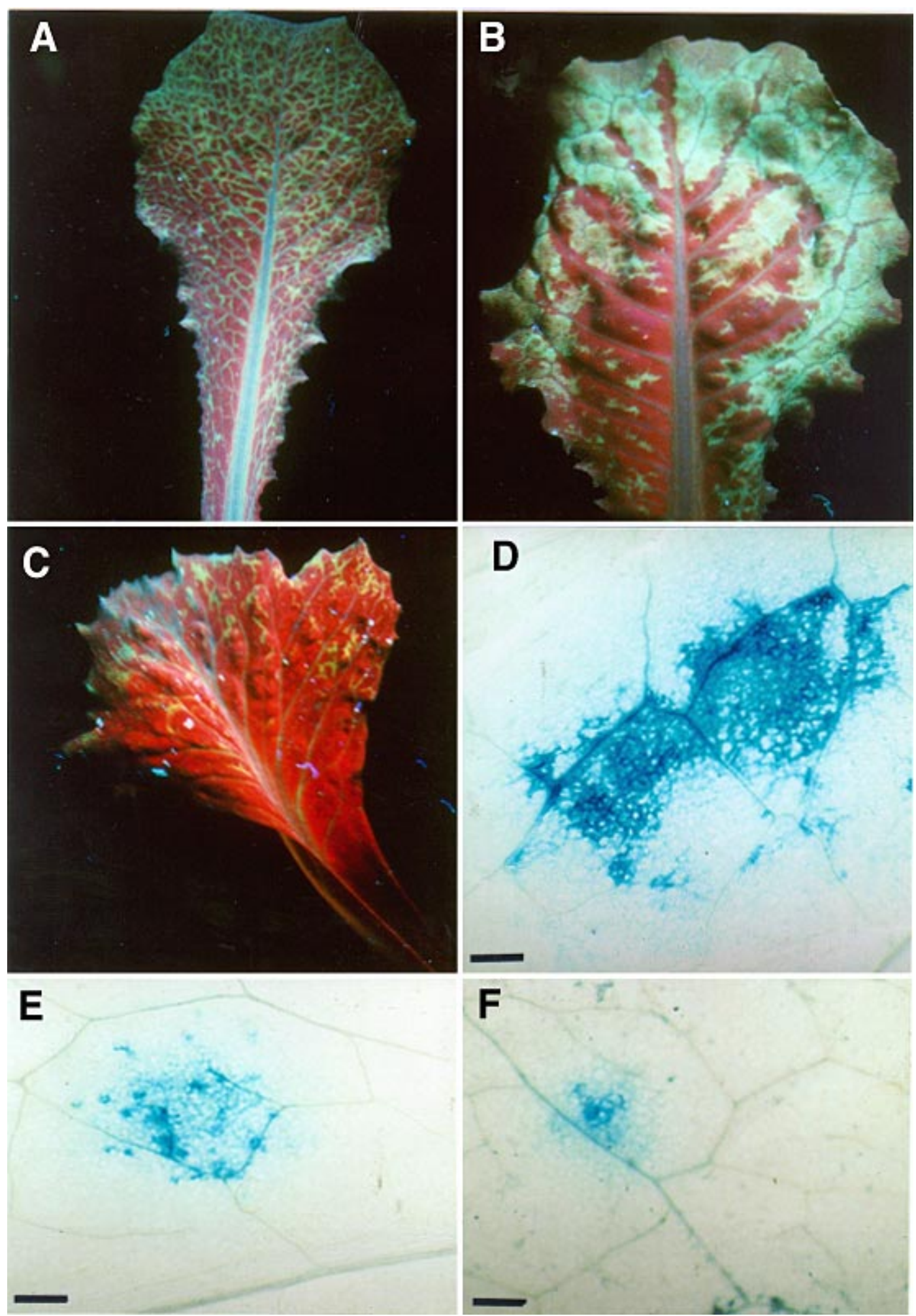

Fig. 4. Visualization under UV light of green fluorescent protein (GFP) fluorescence in LMV-E (Lettuce mosaic virus resistance-breaking isolate)-GFPinfected lettuce (cvs. Trocadero or Vanguard 75) and visualization by $\beta$-glucuronidase (GUS) histochemical staining of infection foci of LMV-E-GUS in inoculated leaves of lettuce cvs. Vanguard or Vanguard 75. A, Fluorescence limited to vicinity of veins in cv. Trocadero at 8 days post inoculation (dpi). Observed fluorescence is superimposable to the vein clearing symptoms caused by the virus. B, Fluorescence expanding to leaf blade of cv. Trocadero at 15 dpi. Again, fluorescence is superimposable to yellow-green areas of the mosaic pattern. C, Representative example of occasional detection of GFP fluorescence in systemically infected leaves of cv. Vanguard 75. Picture was taken at 6 weeks post inoculation. D, Infection foci on an inoculated leaf of cv. Vanguard 75 at 13 dpi. E, Infection foci on an inoculated leaf of cv. Vanguard at $48 \mathrm{~h}$ post inoculation (hpi). F, Infection foci on an inoculated leaf of cv. Vanguard 75 at 48 hpi. Bars $=200 \mu \mathrm{m}$. 


\section{DISCUSSION}

Reporter genes, and especially GFP and GUS, have been incorporated with success into several viral genomes and used as markers of viral infection (Oparka et al. 1997). In the case of potyviruses, the seminal work of Dolja et al. (1992) demonstrated the viability of a recombinant TEV engineered to express a GUS reporter protein as a fusion with the $\mathrm{N}$ terminus of the HC-Pro and thus the feasibility of tagging some potyviral genomes. However, Guo et al. (1998) showed that the recombinant potyvirus PPV-GUS was viable only if an artificial cleavage site specific for the NIa viral proteinase was introduced between the GUS and HC-Pro protein.

In this study it is shown that both GFP and GUS proteins can be fused to the HC-Pro of LMV-E without significantly affecting the viability of the tagged viruses in susceptible cultivars of lettuce. The GFP-tagged virus appears to be very stable since monthly passage for up to 6 months failed to initiate the accumulation of deletion variants having lost the introduced gene. However, prolonged infection of the same host for 4 months led to the appearance of such variants, demonstrating that the GFP-tagged LMV-E is not completely stable. By comparison, the GUS-tagged virus proved to be somewhat less stable, with deletions appearing sooner in the propagated viral populations. These results, as well as the general structure of the deleted genomes recovered, are essentially similar to those reported by Dolja et al. (1993) for TEV-GUS, with two exceptions. First, the stability of the TEV-GUS tagged virus appears to be even lower than that of LMV-E-GUS, deletion mutants of TEV-GUS being almost always recovered following a 3- to 4-week infection period (Dolja et al. 1993). Second, we found that three out of five deletion endpoints clustered near nucleotide 325 of the LMV HC-Pro coding sequence (amino acids 108 to 109), suggesting that this region behaved as a kind of deletion "hot spot," a clustering not observed by Dolja et al. (1993) in TEV-GUS.

The fact that all LMV-E deletion mutants were able to systemically invade susceptible lettuce plants confirms that at least the N-terminal 109 amino acids of the HC-Pro protein

Table 1. Accumulation of LMV-E-GUS and LMV-E-GFPa

\begin{tabular}{|c|c|c|c|c|c|c|c|}
\hline \multirow[b]{3}{*}{ Tag } & \multicolumn{7}{|c|}{ Cultivar and dpi } \\
\hline & \multicolumn{2}{|c|}{ Trocadero } & \multicolumn{3}{|c|}{ Vanguard 75} & \multicolumn{2}{|c|}{ Vanguard } \\
\hline & 15 & 30 & 15 & 30 & 60 & 15 & 30 \\
\hline $\begin{array}{l}\text { LMV- } \\
\text { E-GFP }\end{array}$ & & & $\mathrm{ND}^{\mathrm{b}}$ & ND & $\begin{array}{l}\text { ND or } \\
13 \pm 1^{\mathrm{c}}\end{array}$ & & \\
\hline $\begin{array}{l}\text { LMV- } \\
\text { E-GUS }\end{array}$ & $26 \pm 7$ & $6 \pm 3$ & ND & ND & ND & $2 \pm 0.2$ & $7 *$ \\
\hline \multicolumn{8}{|c|}{ 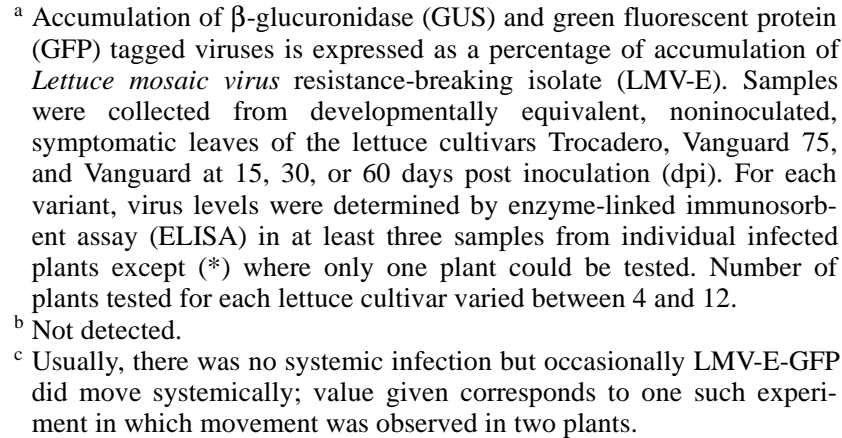 } \\
\hline
\end{tabular}

are not essential for LMV replication and lettuce invasion. These results confirm those obtained for TEV by Dolja et al. $(1993,1997)$ and even increase the dispensable sequence in HC-Pro by 3 amino acids, as seen on a sequence alignment between TEV and LMV (not shown). However, these results differ from those of Guo et al. (1998), who showed that PPVGUS deletions never affected the HC-Pro coding region, suggesting that the $\mathrm{N}$ terminus of the HC-Pro may be essential for PPV viability.

The stability of the GFP- and GUS-tagged viruses makes them excellent candidates for the study of long-term infection processes such as seed or pollen transmission. Sudarshana et al. (1998) used such a GFP reporter system to follow the viral infection process of Bean dwarf mosaic virus from the seedling stage throughout the entire plant's life cycle, and showed that this virus infected flower, pod, and seed teguments in common bean. Our preliminary results show that GUS or GFP reporter activity can be detected in the floral parts of infected lettuce or pea plants (S. German-Retana, T. Candresse, O. Le Gall, and E. Redondo, unpublished).

One surprising finding was that, in addition to generally lowering the viral fitness, tagging of the LMV-E genome affected the viral pathogenicity and the ability of the virus to overcome the $\mathrm{mol}^{2}$ resistance gene carried by the Vanguard 75 cultivar. This effect was macroscopically characterized by the failure, contrary to wild-type LMV-E, to induce symptoms in Vanguard 75, and by the erratic, delayed, and reduced ability of the tagged viruses to systemically invade the resistant cultivar. Currently, we cannot rule out the possibility that the systemic movement of LMV-E-GFP occasionally observed in Vanguard 75 could in fact be due to cell-to cell movement in stem tissues. This negative effect of the tagging on resistance breaking came as a surprise in view of recent findings implicating the $3^{\prime}$ half of the genome of LMV in the breaking of the

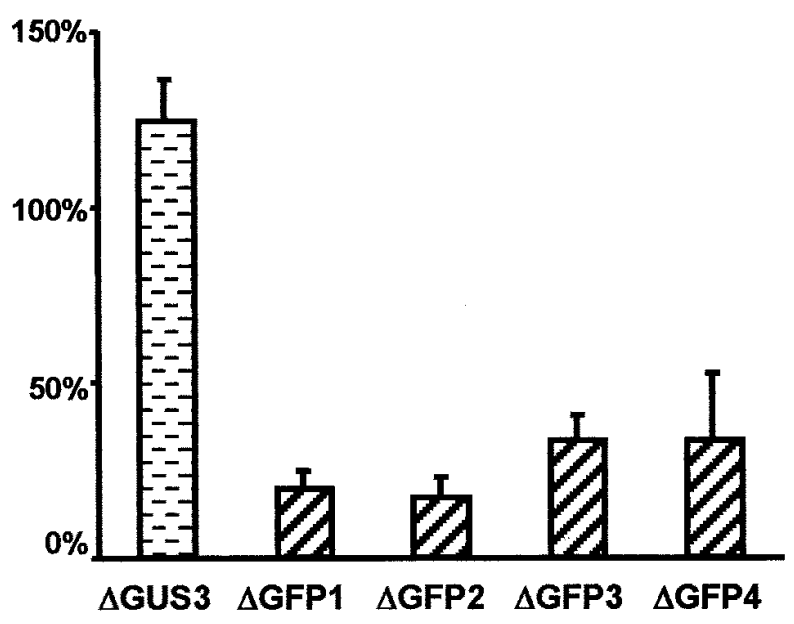

Fig. 5. Accumulation of the green fluorescent protein (GFP) and $\beta$ glucuronidase (GUS) deletion mutants in Trocadero plants. For each deletion mutant, accumulation is expressed as percentage of accumulation of tagged virus from which it is derived. Levels of LMV-E (Lettuce mosaic virus resistance-breaking isolate)-GFP deletion mutants were determined by semiquantitative enzyme-linked immunosorbent assay (ELISA) in three samples from individual plants while $\Delta$ GUS 3 level was determined in four samples from individual plants. All samples were collected from developmentally equivalent, noninoculated, symptomatic leaves at 30 days post inoculation. 
mol resistance genes (E. Redondo, O. Le Gall, and T. Candresse, unpublished) and the fact that the behavior of TEV toward the V20 resistant tobacco variety was not affected by GUS tagging (Schaad and Carrington 1996).

Analysis of the behavior of the deletion variants derived from the tagged viruses indicated that they too were severely affected in their ability to move systemically or to induce symptoms in Vanguard 75, indicating that deletion of 24 amino acids of the HC-Pro $\mathrm{N}$ terminus (LMV-E- $\Delta \mathrm{GFP} 1$ ) is sufficient to cause this effect. Histochemical staining of LMVE-GUS-inoculated leaves showed that the recombinant virus is still able to move cell to cell in Vanguard 75, although at a reduced rate, compared with the situation in the susceptible variety Vanguard. A similar analysis could not be performed for LMV-E-GFP due to the high background fluorescence induced in lettuce leaves by the inoculation procedure. However, the occasional systemic movement of the tagged virus observed in Vanguard 75 indicates that, like LMV-E-GUS, LMV-E-GFP is still able to replicate and move cell to cell in the resistant cultivar.

Two hypotheses can be proposed to explain the observed inhibition of systemic movement of the tagged viruses. The general reduction in fitness observed for the tagged viruses (reduced ability to replicate, accumulate, and move cell to cell) could culminate in a partial block in the viral systemic movement. The alternate hypothesis, consistent with the known involvement of the HC-Pro region in long-distance movement in potyviruses (Cronin et al. 1995), is that the tagging of the HC-Pro specifically affected its ability to perform a function crucial for systemic movement in the resistant host. To the best of our knowledge, these results represent the first evidence for a host genotype-specific effect of modifications of the HC-Pro protein on the ability of a virus to move long distance. Further work is clearly needed to gain a better understanding of the mechanisms involved and to distinguish between these two hypotheses.

\section{MATERIALS AND METHODS}

\section{Plasmid constructions.}

The plasmid p70SLMVE contains an infectious, full-length cDNA copy of LMV-E RNA under the control of the enhanced CaMV 35S promoter and of the NOS terminator (Yang et al. 1998). For simplicity, it is referred to as pLMV-E throughout the text. The plasmid pBIN-m-gfp5-ER was a generous gift from J. Haseloff (MCR, Laboratory of Molecular Biology, Cambridge, UK) and contains the gene $m$-gfp5-ER, encoding a modified Aequorea victoria GFP with better expression and fluorescence in plants (Kirby et al. 1996). This plasmid was used to amplify the GFP gene and introduce flanking AatII and SmaI sites at both ends of the GFP sequence. Since the GFP was to be expressed as a fusion to the HC-Pro of LMV, the initiation methionine was preserved but the termination codon removed during amplification. Following AatII digestion, the amplification product was then incorporated into the AatII site of plasmid pJKK45, which contains nucleotide positions 1 to 2389 of LMV-E cDNA, to give plasmid pJKK45gfp. The AatII site at position 1419 in LMV cDNA, which was used for cloning, corresponds to the second and third codons of the HC-Pro coding sequence. The fused LMV cDNA sequence was inserted into pLMV-E as an RsrII-
BstEII fragment (LMV nucleotides 386 to 2375). The plasmid obtained was named pLMVE-GFP. Following maturation of the HC-Pro $\mathrm{N}$ terminus by the P1 proteinase, the GFP fusion protein expressed should contain the first 3 amino acids of the HC-Pro, a Pro-Gly dipeptide corresponding to the SmaI site, the entire GFP coding sequence, another Pro-Gly dipeptide, and finally the entire sequence of the HC-Pro except for the very first amino acid (Fig. 1). An identical strategy was used to construct a GUS-tagged LMV-E, with the exception that no SmaI sites were introduced and that the methionine initiation codon was removed during the initial GUS gene amplification (Fig. 1). The resulting plasmid was named pLMVE-GUS.

\section{Inoculation of plants.}

LMV isolates were propagated under containment glasshouse conditions on lettuce (Lactuca sativa) plants of cvs. Trocadero, Vanguard (both with no known resistance gene), or Vanguard 75 (harboring the $\mathrm{mol}^{2}$ resistance gene). Mechanical inoculation of young lettuce plants was performed as previously described (Revers et al. 1997a). When purified recombinant plasmids were used as the inoculum, particle bombardment was conducted with a Helios Gene Gun (BioRad, Ivry sur Seine, France) with 1- $\mu$ m gold microcarriers, also as previously described (Yang et al. 1998). Routinely, 100 ng of purified plasmid was used per inoculation.

\section{GUS assays and detection of green fluorescence.}

Histochemical assays for GUS activity were conducted following a protocol slightly adapted from Jefferson (1987). Vacuum was used to infiltrate the GUS staining solution supplemented with $0.2 \%$ (vol/vol) Triton X-100 in leaf fragments. Development of the colorimetric reaction was routinely performed overnight at $37^{\circ} \mathrm{C}$. The size of the infection entry points on inoculated leaves was estimated with a light microscope as the number of GUS-positive cells counted in a plane disk just below the epidermis.

GFP fluorescence was detected visually in whole plants with a $100 \mathrm{~W}$, hand-held, long-wave UV spot-light (Model B100; UV Products, Upland, CA).

\section{Quantification of viral accumulation.}

The relative viral concentrations were estimated by semiquantitative ELISA with a rabbit anti-LMV polyclonal antiserum, kindly provided by Hervé Lot (INRA-Avignon, France). ELISAs were conducted according to Clark and Adams (1977). Alternatively, in some experiments, the viral RNA was quantitated by dot blot hybridization with a ${ }^{32} \mathrm{P}$-labeled, in vitro transcribed cRNA probe as described by Brault et al. (1993).

\section{RT-PCR analysis of recombinant virus progeny.}

The cDNA spanning the region containing the introduced genes in the recombinant LMV isolates was amplified by RTPCR with crude leaf extracts as the starting material. The first-strand primer (01963M) was complementary to nucleotides 1963 to 1943 within the HC-Pro coding region, while the second-strand primer (01317P) corresponded to nucleotides 1314 to 1331 within the P1 coding region. The size of the amplified products was determined by either agarose or polyacrylamide gel electrophoresis. Following restriction enzyme analysis, amplification products derived from revertant viruses 
having lost the introduced genes were sequenced directly without cloning (Eurogentec, Seraing, Belgium).

\section{ACKNOWLEDGMENTS}

We are grateful to Kathryn Mayo-Candresse for her help with the English of the manuscript, Hervé Lot (INRA-Avignon, France) for the LMV antiserum and very fruitful and stimulating discussions, and Benny Raccah (Volcani Center, Bet Dagan, Israel) for the transmission tests of LMV-E-GFP. This work was partially supported by the EPR Aquitaine.

\section{LITERATURE CITED}

Baulcombe, D. C., Chapman, S., and Santa Cruz, S. 1995. Jellyfish green fluorescent protein as a reporter for virus infection. Plant J. 7: 1045-1053.

Blanc, S., Dolja, V. V., Llave, C., and Pirone, T. P. 1999. Histidinetagging and purification of tobacco etch potyvirus helper component protein. J. Virol. Methods 77:11-15.

Bos, L., Huijberts, N., and Cuperus, C. 1994. Further observations on variation of lettuce mosaic virus in relation to lettuce Lactuca sativa, and a discussion of resistance terminology. Eur. J. Plant Pathol. 100: 293-314.

Brault, V., Candresse, T., Le Gall, O., Delbos, R. P., Lanneau, M., and Dunez, J. 1993. Genetically engineered resistance against grapevine chrome mosaic nepovirus. Plant Mol. Biol. 21:89-97.

Casper, S. J., and Holt, C.A. 1996. Expression of the green fluorescent protein-encoding gene from a tobacco mosaic virus-based vector. Gene 173:69-73.

Chalfie, M., Tu, Y., Euskirchen, G., Ward, W. W., and Prasher, D. C. 1994. Green fluorescent protein as a marker for gene expression. Science 263:802-804.

Clark, M. F., and Adams, A. N. 1977. Characteristics of the microplate method of enzyme-linked immunosorbent assay for the detection of plant viruses. J. Gen. Virol. 34:475-483.

Cronin, S., Verchot, J., Haldemann-Cahill, R., Schaad, M. C., and Carrington, J. C. 1995. Long-distance movement factor: A transport function of the potyvirus helper component-proteinase. Plant Cell 7: 549-559.

Dinant, S., and Lot, H. 1992. Lettuce mosaic virus. Plant Pathol. 41: 528-542.

Dolja, V. V., McBride, H. J., and Carrington, J. C. 1992. Tagging of plant potyvirus replication and movement by insertion of $\beta$-glucuronidase into the viral polyprotein. Proc. Natl. Acad. Sci. USA 89: 10208-10212.
Dolja, V. V., Herndon, K. L., Pirone, T. P., and Carrington, J. C. 1993. Spontaneous mutagenesis of a plant potyvirus genome after insertion of a foreign gene. J. Virol. 67:5968-5975.

Dolja, V. V., Hong, J., Keller, K. E., Martin, R. R., and Peremyslov, V. V. 1997. Suppression of potyvirus infection by coexpressed closterovirus protein. Virology 234:243-252.

Dougherty, W. G., Parks, T. D., Smith, H. A., and Lindbo, A. 1990. Expression of potyvirus genome: The role of proteolytic processing. Pages 124-139 in: Viral Genes and Plant Pathogenesis. SpringerVerlag, Vienna.

Guo, H. S., López-Moya, J. J., and García, J. A. 1998. Susceptibility to recombination rearrangements of a chimeric plum pox potyvirus genome after insertion of a foreign gene. Virus Res. 57:183-195.

Jefferson, R. A. 1987. Assaying chimeric genes in plants: The GUS gene fusion system. Plant Mol. Biol. Rep. 5:387-405.

Kirby, R. S., Golbik, R., Sever, R., and Haseloff, J. 1996. Mutations that suppress the thermosensitivity of green fluorescent protein. Curr. Biol. 6:1653-1663.

Oparka, K. J, Roberts, A. G., Santa-Cruz, S., Boevink, P., Prior, D. A. M., and Smallcombe, A. 1997. Using GFP to study virus invasion and spread in plant tissues. Nature 388:401-402.

Pink, D. A. C., Kostova, D., and Walkey, D. G. A. 1992a. Differentiation of pathotypes of lettuce mosaic virus. Plant Pathol. 41:5-12.

Pink, D. A. C., Lot, H., and Johnson, R. 1992b. Novel pathotypes of lettuce mosaic virus-breakdown of a durable resistance. Euphytica 63: 169-174.

Reichman, J. L., Lain, S., and Garcia, J. A. 1992. Highlights and prospects of potyvirus molecular biology. J. Gen. Virol. 73:1-16.

Revers, F., Lot, H., Souche, S., Le Gall, O., Candresse, T., and Dunez, J. 1997a. Biological and molecular variability of lettuce mosaic virus isolates. Phytopathology 87:397-403.

Revers, F., Yang, S.J., Walter, J., Lot, H., Souche, S., Le Gall, O., Candresse, T., and Dunez, J. 1997b. Comparison of the complete nucleotide sequences of two isolates of lettuce mosaic virus differing in their biological properties. Virus Res. 47:167-177.

Ryder, E. J. 1979. "Vanguard 75" lettuce. HortScience 14:284-286.

Schaad, M. C., and Carrington, J. C. 1996. Suppression of long distance movement of tobacco etch virus in a non-susceptible host. J. Virol. 70:2556-2561

Sudarshana, M. R., Wang, H. L., Lucas, W. J., and Gilbertson, R. L. 1998. Dynamics of bean dwarf mosaic geminivirus cell-to-cell and long-distance movement in Phaseolus vulgaris revealed, using the green fluorescent protein. Mol. Plant-Microbe Interact. 11:277-291.

Yang, S. J., Revers, F., Souche, S., Lot, H., Le Gall, O., Candresse, T., and Dunez, J. 1998. Construction of full-length cDNA clones of lettuce mosaic virus LMV and the effects of intron-insertion on their viability in Escherichia coli, and on their infectivity to plants. Arch. Virol. 143:2443-2451. 